\title{
HAUSDORFF DIMENSION AND LIMITS OF KLEINIAN GROUPS
}

\author{
R.D. Canary and E.C. Taylor
}

\begin{abstract}
In this paper we prove that if $M$ is a compact, hyperbolizable 3manifold, which is not a handlebody, then the Hausdorff dimension of the limit set is continuous in the strong topology on the space of marked hyperbolic 3-manifolds homotopy equivalent to $M$. We similarly observe that for any compact hyperbolizable 3-manifold $M$ (including a handlebody), the bottom of the spectrum of the Laplacian gives a continuous function in the strong topology on the space of topologically tame hyperbolic 3-manifolds homotopy equivalent to $M$.
\end{abstract}

\section{Introduction}

Let $M$ be a compact hyperbolizable 3-manifold and let $\mathcal{D}\left(\pi_{1}(M)\right)$ denote the space of discrete faithful representations of $\pi_{1}(M)$ into $\mathrm{PSL}_{2}(\mathbf{C})$. Given $\rho \in \mathcal{D}\left(\pi_{1}(M)\right)$, let $D(\rho)$ denote the Hausdorff dimension of the limit set of $\rho\left(\pi_{1}(M)\right)$. A sequence $\left\{\rho_{i}\right\}$ in $\mathcal{D}\left(\pi_{1}(M)\right)$ converges to $\rho$ in the strong topology if and only if $\left\{\rho_{i}\right\}$ converges to $\rho$ in the compact-open topology and $\left\{\rho_{i}\left(\pi_{1}(M)\right)\right\}$ converges to $\rho\left(\pi_{1}(M)\right)$ geometrically.

The main result of this paper is the following theorem:

Main Theorem. Let $M$ be a compact, hyperbolizable 3-manifold which is not homeomorphic to a handlebody. Then $D$ is continuous on $\mathcal{D}\left(\pi_{1}(M)\right)$ in the strong topology.

One can also consider the function $\Lambda$ on $\mathcal{D}\left(\pi_{1}(M)\right)$ given by letting $\Lambda(\rho)$ be the bottom $\lambda_{0}\left(N_{\rho}\right)$ of the spectrum of the Laplacian of the quotient manifold $N_{\rho}=\mathbf{H}^{3} / \rho\left(\pi_{1}(M)\right)$ associated to $\rho$. It follows from work of Patterson $[\mathrm{P}]$, Sullivan [S1], Bishop-Jones [BiJo] and Canary $[\mathrm{C}]$, that if $\rho$ is topologically tame, then $\Lambda(\rho)=D(\rho)(2-D(\rho))$ if $D(\rho) \geq 1$ and that $\Lambda(\rho)=1$ otherwise. Let $T T\left(\pi_{1}(M)\right)$ denote the set of topologically

Research of the first author was supported in part by the National Science Foundation and a fellowship from the Sloan Foundation. 
tame representations in $\mathcal{D}\left(\pi_{1}(M)\right)$. Our main theorem will follow from the following result and work of Bishop-Jones [BiJo], Canary [C] and Taylor [T].

Theorem 4.1. Let $M$ be a compact, hyperbolizable 3-manifold. If $\left\{\rho_{i}\right\}$ is a sequence in $\mathcal{D}\left(\pi_{1}(M)\right)$ converging strongly to $\rho$, where $\rho$ is geometrically finite, then $\left\{\Lambda\left(\rho_{i}\right)\right\}$ converges to $\Lambda(\rho)$.

By combining Theorem 4.1 with work of Canary [C] we obtain the following corollary.

Corollary A. Let $M$ be a compact, hyperbolizable 3-manifold. Then $\Lambda$ is continuous on $T T\left(\pi_{1}(M)\right)$ in the strong topology.

The Main Theorem also gives rise to the following characterization of strong convergence in the case when the algebraic limit is geometrically finite.

Corollary B. Let $M$ be a compact hyperbolizable 3-manifold which is not homeomorphic to a handlebody. Suppose that a sequence $\left\{\rho_{i}\right\}$ in $\mathcal{D}\left(\pi_{1}(M)\right)$ converges (in the compact-open topology) to $\rho \in \mathcal{D}\left(\pi_{1}(M)\right)$ such that $\rho$ is geometrically finite. Then $\left\{\rho_{i}\right\}$ converges strongly to $\rho$ if and only if $\left\{D\left(\rho_{i}\right)\right\}$ converges to $D(\rho)$.

It has previously been observed, see Taylor [T], Canary-Minsky [CMi] or Bishop-Jones [BiJo], that $D$ and $\Lambda$ are not continuous in the compact-open (or algebraic) topology on $\mathcal{D}\left(\pi_{1}(M)\right)$. Canary and Minsky [CMi] proved that $\Lambda$ is continuous on the subset of purely hyperbolic representations in $T T\left(\pi_{1}(M)\right)$ in the algebraic and the strong topologies. McMullen [Mc] has recently proven that $D$ is not continuous in the strong topology on $\mathcal{D}\left(\pi_{1}(M)\right)$ if $M$ is a handlebody.

Bonahon [Bo] proved that $\mathcal{D}\left(\pi_{1}(M)\right)=T T\left(\pi_{1}(M)\right)$ if $\pi_{1}(M)$ is freely indecomposable. It is conjectured that $T T\left(\pi_{1}(M)\right)$ always equals $\mathcal{D}\left(\pi_{1}(M)\right)$ (see Marden $[\mathrm{M}]$.$) One conjectures that \Lambda$ is always continuous on $\mathcal{D}\left(\pi_{1}(M)\right)$ in the strong topology.

McMullen $[\mathrm{Mc}]$ has independently proven a generalization of Theorem 4.1 and a variety of interesting related results. His proof makes use of the language of Patterson-Sullivan measures, while ours uses spectral theory. Related results have also been obtained by Comar-Taylor [ComT].

Acknowledgements. The authors would like to thank the University of Southampton and EPSRC for their support. 


\section{Preliminaries}

In this section, we recall the definitions of some of the terms used in the introduction and introduce some necessary background material.

A compact, orientable 3-manifold is called hyperbolizable if there exists a complete hyperbolic 3-manifold homeomorphic to the interior of $M$. We will assume throughout the remainder of the paper that $\pi_{1}(M)$ is non-abelian. (If $\pi_{1}(M)$ is abelian, then both $\Lambda$ and $D$ are constant on $\mathcal{D}\left(\pi_{1}(M)\right)$.)

A Kleinian group is a discrete subgroup of $\mathrm{PSL}_{2}(\mathbf{C})$, which we regard both as the group of conformal automorphisms of the Riemann sphere and as the group of orientation-preserving isometries of $\mathbf{H}^{3}$. A sequence $\left\{\Gamma_{i}\right\}$ of Kleinian groups is said to converge geometrically to a Kleinian group $\Gamma$ if every element $\gamma \in \Gamma$ is a limit of a sequence $\left\{\gamma_{i} \in \Gamma_{i}\right\}$, and every accumulation point of such a sequence lies in $\Gamma$. A sequence $\left\{\rho_{i}\right\}$ in $\mathcal{D}\left(\pi_{1}(M)\right)$ converges strongly to $\rho$ if $\left\{\rho_{i}\right\}$ converges to $\rho$ in the compactopen topology and $\left\{\rho_{i}\left(\pi_{1}(M)\right)\right\}$ converges geometrically to $\rho\left(\pi_{1}(M)\right)$. The resulting topology on $\mathcal{D}\left(\pi_{1}(M)\right)$ is called the strong topology. (One may explicitly exhibit a basis for the strong topology on $\mathcal{D}\left(\pi_{1}(M)\right)$ by combining open sets in the compact-open topology and open sets coming from the Chabauty, or geometric, topology on the space of all Kleinian groups, see section 3.1 of Canary-Epstein-Green [CEGr]. Since the resulting topology is Hausdorff and first countable it will always suffice to discuss sequences.)

The limit set $L(\Gamma)$ of a Kleinian group $\Gamma$ (which is not virtually abelian) is defined to be the smallest non-empty, closed subset of the Riemann sphere which is invariant under $\Gamma$. We define a function $D$ on $\mathcal{D}\left(\pi_{1}(M)\right)$ by letting $D(\rho)$ denote the Hausdorff dimension of the limit set $L\left(\rho\left(\pi_{1}(M)\right)\right.$ of $\rho\left(\pi_{1}(M)\right)$.

If $N$ is a complete hyperbolic 3-manifold, we let $\lambda_{0}(N)=\inf \operatorname{spec}(-\Delta)$ where $\Delta=\operatorname{div}(\operatorname{grad})$ is the Laplacian. We define the function $\Lambda$ on $\mathcal{D}\left(\pi_{1}(M)\right)$ by setting $\Lambda(\rho)=\lambda_{0}\left(N_{\rho}\right)$ where $N_{\rho}=\mathbf{H}^{3} / \rho\left(\pi_{1}(M)\right)$.

The convex core $C(N)$ of a hyperbolic 3-manifold $N=\mathbf{H}^{3} / \Gamma$ is defined to be the quotient of the convex hull $C H(L(\Gamma))$ of the limit set $L(\Gamma)$ by $\Gamma$. Given any $K \geq 0$, let $C_{K}(N)$ denote the closed metric $K$-neighborhood of $C(N)$. There is a retraction $r_{K}$ of $N$ onto $C_{K}(N)$ given by taking any point in $N$ to the unique point in $C_{K}(N)$ nearest to it. (This retraction is discussed extensively in Epstein-Marden [EM].)

A hyperbolic 3-manifold $N$ is said to be geometrically finite if $C_{K}(N)$ has finite volume for all $K \geq 0 . \quad N$ is said to be topologically tame if it is homeomorphic to the interior of a compact manifold. We will call a 
representation $\rho \in \mathcal{D}\left(\pi_{1}(M)\right)$ geometrically finite (or topologically tame) if $N_{\rho}=\mathbf{H}^{3} / \rho\left(\pi_{1}(M)\right)$ is geometrically finite (or topologically tame).

If $N$ is a hyperbolic 3-manifold then $\lambda_{0}(N) \leq \lambda_{0}\left(\mathbf{H}^{3}\right)=1$. If $\lambda_{0}(N)<1$ and $N$ is geometrically finite, then there exists a unique $C^{\infty}$ positive eigenfunction $\phi$ of $-\Delta$ on $N$ with $L^{2}$-norm 1 and the eigenvalue of $\phi$ is $\lambda_{0}(N)$ (see Lax-Phillips [LPh] or Sullivan [S2].) When it exists, we call $\phi$ the normalized first eigenfunction. If $N$ is geometrically finite and $\lambda_{0}(N)=1$, then $N$ does not carry any eigenfunctions of $-\Delta$ which lie in $L^{2}(N)$.

Patterson and Sullivan first observed that there is a deep relationship between $D$ and $\Lambda$ if $\rho$ is geometrically finite. In deriving the Main Theorem from Theorem 4.1 we will use the following theorem, whose statement combines results of Patterson [P], Sullivan [S1], Canary [C] and BishopJones [BiJo].

Theorem 2.1. Let $M$ be a compact hyperbolizable 3-manifold. If $\rho \in$ $T T\left(\pi_{1}(M)\right)$ then $\Lambda(\rho)=D(\rho)(2-D(\rho))$ if $D(\rho) \geq 1$, while $\Lambda(\rho)=1$ otherwise.

One may combine the main results of Canary [C] and Bishop-Jones [BiJo] to show that the volume of the convex core of a hyperbolic 3-manifold provides a lower bound for the Hausdorff dimension of its limit set.

Theorem 2.2. Let $M$ be a compact hyperbolizable 3-manifold. There exists a constant $K$ so that if $\rho \in \mathcal{D}\left(\pi_{1}(M)\right)$, then $D(\rho) \geq 2-\frac{K}{\operatorname{vol}\left(C\left(N_{\rho}\right)\right)}$.

Proof of 2.2. If $\operatorname{vol}\left(C\left(N_{\rho}\right)\right)$ is infinite, then $N_{\rho}$ is geometrically infinite, so the main theorem of Bishop and Jones [BiJo] implies that $D(\rho)=2$. If $\operatorname{vol}\left(C\left(N_{\rho}\right)\right)$ is finite, then the main result of Canary [C] (see also Corollary B of Burger-Canary $[\mathrm{BuC}])$ implies that $D(\rho) \geq 2-\frac{4 \pi\left|\chi\left(\partial C\left(N_{\rho}\right)\right)\right|}{\operatorname{vol}\left(C\left(N_{\rho}\right)\right)}$ where $\chi\left(\partial C\left(N_{\rho}\right)\right)$ denote the Euler characteristic of the boundary of $C\left(N_{\rho}\right)$. Since $\chi\left(\partial C\left(N_{\rho}\right)\right)=\chi(\partial M)$ if $N_{\rho}$ is geometrically finite (see Bowditch [Bow]), the theorem holds with $K=4 \pi|\chi(\partial M)|$.

It is useful to divide a hyperbolic 3-manifold $N$ up into thick and thin parts. Given $\epsilon>0$ we define $N_{\operatorname{thin}(\epsilon)}$ to be the set of points in $N$ with injectivity radius at most $\epsilon$ and $N_{\operatorname{thick}(\epsilon)}$ to be the set of points with injectivity radius at least $\epsilon$. The Margulis lemma (see Benedetti-Petronio [BPe] for example) implies that there exists $\mathcal{M}_{3}>0$ such that if $\epsilon<\mathcal{M}_{3}$ then every component of $N_{\operatorname{thin}(\epsilon)}$ is either a solid torus neighborhood of a closed geodesic or the quotient of a horoball in $\mathbf{H}^{3}$ by a discrete group of parabolic elements preserving the horoball. If $T$ is a component of $N_{\operatorname{thin}(\epsilon)}$ we let $\mathcal{S}(T)$ denote the neighborhood of radius 1 of $\partial T$ in $T$. We will often 
consider the submanifold $N_{\epsilon}^{S}$ which is taken to be $N_{\text {thick }(\epsilon)} \cup \mathcal{S}\left(N_{\operatorname{thin}(\epsilon)}\right)$.

Outline of paper. In the next two sections we will assemble the proof of Theorem 4.1 (which asserts that the lowest eigenvalue of the Laplacian is continuous on a strongly convergent sequence with a geometrically finite limit) and in section 5 we observe that the Main Theorem and its corollaries follow from Theorem 4.1 and previously known results. In a final section we describe a conjecture concerning the relationship between geometric convergence and the behavior of $\Lambda$.

As the next section is spent proving the technical lemmas used in the proof of Theorem 4.1, we will provide a brief outline of the proof here. A theorem of Taylor [T] insures us, since $N_{\rho}$ is geometrically finite, that we may assume that $N_{i}=N_{\rho_{i}}$ is geometrically finite for all $i$. Since $\Lambda$ is upper semicontinuous, we can reduce to the case that $\lim \lambda_{0}\left(N_{i}\right)$ exists and is less than 1. Let $\left\{\phi_{i}\right\}$ be the sequence of normalized first eigenfunctions associated to $\left\{\rho_{i}\right\}$. We observe that most of the support of each $\phi_{i}$ lies on a definite neighborhood $C_{K}\left(N_{i}\right)$ of the convex core (see Lemma 3.5)) and that a definite portion of the support of each $\phi_{i}$ lies in the thick part $\left(N_{i}\right)_{\epsilon}^{S}$ (see Lemma 3.3.) It then follows that there is a definite portion of the support contained in $C_{K}\left(N_{i}\right) \cap\left(N_{i}\right)_{\epsilon}^{S}$ for all $i$. We then show (see Lemma 3.6) that there is a definite bound on the diameter, and hence the volume, of $C_{K}\left(N_{i}\right) \cap\left(N_{i}\right)_{\epsilon}^{S}$. Putting this together we find a point in each $N_{i}$ at which $\phi_{i}$ is at least some definite value. This will imply that the limit of a subsequence of $\left\{\phi_{i}\right\}$ is a non-zero, positive, $L^{2}$ eigenfunction of $-\Delta$ on $N_{\rho}$ whose eigenvalue is equal to $\lim \lambda_{0}\left(N_{i}\right)$, and hence that $\lambda_{0}(N)=\lim \lambda_{0}\left(N_{i}\right)$.

\section{The Lemmas}

We first prove that if $N$ is geometrically finite and $\lambda_{0}(N) \neq 1$, then some definite proportion of the normalized eigenfunction is supported on the thick part of the manifold. The proof relies on the following generalization of Lemma 2 in Dodziuk-Randol [DR]. The proof of Lemma 3.1, which relies on the fact that if $T$ is a component of $N_{\operatorname{thin}(\epsilon)}$ then $\lambda_{0}(T) \geq 1$, is the same as that of Lemma 2 in [DR].

Lemma 3.1 (Dodziuk-Randol [DR]). Let $0<\epsilon<\mathcal{M}_{3}$ and $\delta>0$. There exists a constant $\alpha>0$ such that if $N$ is a hyperbolic 3-manifold, $T$ is a component of $N_{\operatorname{thin}(\epsilon)}, T \neq \mathcal{S}(T)$, and $f \in C^{1}(N) \cap L^{2}(N)$ such that

1. $\int_{T} f^{2} \geq c$,

2. $\quad \int_{\mathcal{S}(T)}|\nabla f|^{2} \leq \alpha c$, and 
3. $\int_{\mathcal{S}(T)} f^{2} \leq \alpha c$, then

$$
\int_{T}|\nabla f|^{2} \geq(1-\delta) c
$$

We will also make repeated use of Yau's Harnack inequality (see [Y]) which we state in the restricted setting in which we use it.

Lemma 3.2 (Yau's Harnack Inequality). There exists a constant $R$ such that if $N$ is a complete hyperbolic 3-manifold and $\phi$ is a positive $C^{\infty}$-eigenfunction of $-\Delta$ with eigenvalue between 0 and 1 , then $|\nabla \phi(x)| \leq R \phi(x)$ for all $x \in N$.

We are now prepared to prove that the $L^{2}$-norm of the restriction of the normalized first eigenfunction to the thick part is bounded from below.

Lemma 3.3. Given $0<\epsilon<\mathcal{M}_{3}$ and $\delta_{1}>0$, there exists $C>0$ such that if $N$ is a geometrically finite hyperbolic 3-manifold, $\lambda_{0}(N) \leq 1-\delta_{1}$, and $\phi$ is its normalized first eigenfunction, then

$$
\int_{N_{\epsilon}^{S}} \phi^{2} \geq C
$$

Proof of 3.3. Since $\lambda_{0}(N) \geq 0, \delta_{1} \leq 1$. Lemma 3.1 assures that we can choose $\alpha>0$ such that if $f \in \bar{C}^{1}(N) \cap L^{2}(N), \int_{N_{\text {thin }(\epsilon)}} f^{2} \geq c$, $\int_{\mathcal{S}\left(N_{\text {thin }(\epsilon)}\right)}|\nabla f|^{2} \leq \alpha c$, and $\int_{\mathcal{S}\left(N_{\operatorname{thin}(\epsilon))}\right)} f^{2} \leq \alpha c$, then $\int_{N_{\text {thin }(\epsilon)}}|\nabla f|^{2} \geq\left(1-\frac{\delta_{1}}{2}\right) c$. Let $C=\min =\left\{\frac{\delta_{1}}{2}, \frac{\alpha}{2 R^{2}}\right\}$ where $R$ is the constant in Yau's Harnack inequality. If $\int_{N_{\epsilon}^{S}} \phi^{2} \leq C$, then the above inequalities hold with $c=1-\frac{\delta_{1}}{2}$. Therefore,

$$
\int_{N_{\text {thin }(\epsilon)}}|\nabla \phi|^{2} \geq\left(1-\frac{\delta_{1}}{2}\right)^{2}>1-\delta_{1} \geq \lambda_{0}(N)
$$

which contradicts the fact that $\int_{N}|\nabla \phi|^{2}=\lambda_{0}(N)$. This establishes our claim.

We next observe that a definite portion of the support of the normalized first eigenfunction of a geometrically finite hyperbolic 3-manifold lies in a definite radius neighborhood of the convex core. We first obtain the following generalization of Proposition 4.2 in Burger-Canary [BuC] which asserts that outside a definite neighborhood of the convex core the normalized first eigenfunction decays exponentially at a definite rate.

Lemma 3.4. Given $\delta_{1}>0$ there exists $B>0$ and $\delta_{2}>0$ such that if $N$ is a geometrically finite hyperbolic 3-manifold with $\lambda_{0}(N)<1-\delta_{1}$, 
$\phi$ is its normalized first eigenfunction, and $d(x, C(N))>B$, then $\phi(x) \leq e^{-\left(1+\delta_{2}\right) d\left(x, r_{B}(x)\right)} \phi\left(r_{B}(x)\right)$.

Proof of 3.4. Let $N=\mathbf{H}^{3} / \Gamma$. We will work in the ball model for $\mathbf{H}^{3}$. Given $\xi \in S^{2}=\partial \mathbf{H}^{3}$, let $f_{\xi}: \mathbf{H}^{3} \rightarrow \mathbf{R}$ be given by $f_{\xi}(x)=\frac{1-|x|^{2}}{|x-\xi|^{2}}$. For any $x \in \mathbf{H}^{3}$, the geodesic ray determined by $\nabla f_{\xi}(x)$ ends at $\xi$ and $\left|\nabla f_{\xi}(x)\right|=f_{\xi}(x)$ for all $x$.

Patterson and Sullivan (see [S2]) proved that if $N$ is geometrically finite, $\lambda_{0}(N)<1$ and $\phi$ is its normalized first eigenfunction, then there exists a measure $\mu$ on $S^{2}$ which is supported on the limit set of $\Gamma$ such that if we define

$$
\widetilde{\phi}(x)=\int_{S^{2}}\left(f_{\xi}(x)\right)^{\delta(N)} d \mu,
$$

for all $x \in \mathbf{H}^{3}$ (where $\lambda_{0}(N)=\delta(N)(2-\delta(N)$ ) and $\delta(N)>1$ ), then $\widetilde{\phi}$ descends to the map $\phi$. In particular, there exists $1>d>0$ such that if $\lambda_{0}(N)<1-\delta_{1}$, then $\delta(N)>1+d$.

We may choose $B$ large enough so that if $y$ lies on the positive portion of the $x_{3}$-axis and $d(0, y) \geq B$, then if $u$ and $v$ are any two unit vectors in $T_{y}\left(\mathbf{H}^{3}\right)$ whose associated geodesic rays end in the southern hemisphere then $u \cdot v \geq 1-\frac{d}{2}$.

We now claim that if we choose $B$ in this manner then the above statement is true with $\delta_{2}=\frac{d}{2}-\frac{d^{2}}{2}$. Given $x \in N-C_{B}(N)$, let $x^{\prime}$ denote a point in the preimage of $x$ in $\mathbf{H}^{3}$. We may normalize so that $x^{\prime}$ lies on the positive $x_{3}$-axis and $\tilde{r}_{0}\left(x^{\prime}\right)=0$ (where $\tilde{r}_{K}$ is the nearest point retraction of $\mathbf{H}^{3}$ onto the closed neighborhood of radius $K$ of $\left.C H(L(\Gamma))\right)$. In this normalization, $L(\Gamma)$ lies entirely in the southern hemisphere.

If $y$ is a point on the $x_{3}$-axis between $x^{\prime}$ and $\tilde{r}_{B}\left(x^{\prime}\right)$ and $\xi \in L(\Gamma)$, then

$$
\nabla\left(f_{\xi}(y)^{\delta(N)}\right) \cdot \vec{u}_{y} \geq(1+d)\left(1-\frac{d}{2}\right) f_{\xi}(y)^{\delta(N)}=\left(1+\delta_{2}\right) f_{\xi}(y)^{\delta(N)}
$$

where $\vec{u}_{y}$ is the unit vector in $T_{y}\left(\mathbf{H}^{3}\right)$ pointing towards the origin. Therefore,

$$
\nabla \widetilde{\phi}(y) \cdot \vec{u}_{y} \geq\left(1+\delta_{2}\right) \widetilde{\phi}(y) .
$$

We then establish the desired inequality by integrating $\nabla \widetilde{\phi}$ along the positive $x_{3}$-axis from $x^{\prime}$ to $\tilde{r}_{B}\left(x^{\prime}\right)$ and noticing that $\tilde{r}_{B}\left(x^{\prime}\right)$ covers $r_{B}(x)$.

We use this to prove

Lemma 3.5. Given $\delta_{1}>0$ and $\delta_{3}>0$, there exists $K>0$ such that if $N$ is a geometrically finite hyperbolic 3-manifold, $\lambda_{0}(N) \leq 1-\delta_{1}$, and $\phi$ is its 
normalized first eigenfunction then

$$
\int_{C_{K}(N)} \phi^{2} \geq 1-\delta_{3}
$$

Proof of 3.5. Choose $B$ and $\delta_{2}$ as given by Lemma 3.4. Let $h: N-C_{B}(N)$ $\rightarrow \partial C_{B}(N) \times(0, \infty)$ be given by $h(x)=\left(r_{B}(x), d\left(x, r_{B}(x)\right)\right.$. As in the proof of Theorem 2.3.1 of Epstein-Marden [EM], we see that $h$ is Lipschitz and that there exist constants $D>0$ and $D^{\prime}>0$ so that

$$
D e^{2 d\left(x, r_{B}(x)\right)} \leq \frac{1}{|d h(x)|} \leq D^{\prime} e^{2 d\left(x, r_{B}(x)\right)}
$$

almost everywhere. Therefore, for all $T>S \geq 0$,

$$
\int_{C_{B+T}(N)-C_{B+S}(N)} \phi^{2} \leq D^{\prime} \int_{\partial C_{B}(N)} \int_{S}^{T} e^{-2 \delta_{2} t} \phi^{2}(x) d A d t
$$

(where $d A$ denotes the area measure on $\partial C_{B}(N)$ ) which implies that

$$
\int_{C_{B+T}(N)-C_{B+S}(N)} \phi^{2} \leq \frac{D^{\prime}}{2 \delta_{2}}\left(e^{-2 \delta_{2} S}-e^{-2 \delta_{2} T}\right) \int_{\partial C_{B}(N)} \phi^{2} d A .
$$

On the other hand, applying Yau's Harnack inequality we see that if $x \in$ $N-C_{B}(N)$, then $\phi(x) \geq e^{-R d\left(x, r_{B}(x)\right)} \phi\left(r_{B}(x)\right)$. Hence,

$$
\begin{aligned}
\int_{C_{B+1}(N)-C_{B}(N)} \phi^{2} & \geq D \int_{\partial C_{B}(N)} \int_{0}^{1} e^{-2 R t} \phi^{2}(x) d A d t \\
& \geq \frac{D}{2 R}\left(1-e^{-2 R}\right) \int_{\partial C_{B}(N)} \phi^{2} d A .
\end{aligned}
$$

Since, $\int_{C_{B+1}(N)-C_{B}(N)} \phi^{2} \leq 1$, we see that

$$
\int_{\partial C_{B}(N)} \phi^{2} d A \leq \frac{2 R}{D\left(1-e^{-2 R}\right)} .
$$

Therefore, if we set

$$
S=\frac{\log \left(D^{\prime} R\right)-\log \left(\delta_{2} \delta_{3} D\left(1-e^{-2 R}\right)\right)}{2 \delta_{2}},
$$

then

$$
\int_{N-C_{B+S}(N)} \phi^{2} \leq \delta_{3}
$$

Let $K=B+S$ and the claim is established.

We now need to know some basic facts about the convergence of the convex cores in our sequence. The following argument is a generalization of arguments given in Taylor's paper [T]. 
Lemma 3.6. Let $M$ be a compact hyperbolizable 3-manifold, $0<\epsilon<\mathcal{M}_{3}$ and $K>0$. Let $\left\{\rho_{i}\right\}$ be a sequence of representations in $\mathcal{D}\left(\pi_{1}(M)\right)$ converging strongly to a geometrically finite representation $\rho$. Then there exists a constant $A>0$ such that the diameter of $\left(N_{i}\right)_{\epsilon}^{S} \cap C_{K}\left(N_{i}\right)$ is less than $A$ for all sufficiently large $i$.

In the proof of Lemma 3.6, and later in the proof of Theorem 4.1, we will make use of the following alternative characterization of geometric convergence (see Canary-Epstein-Green [CEGr] or Benedetti-Petronio [BPe] for a proof.)

Lemma 3.7. Let $\left\{\Gamma_{i}\right\}$ be a sequence of Kleinian groups converging geometrically to $\Gamma$. Let $N_{i}=\mathbf{H}^{3} / \Gamma_{i}$ and $N=\mathbf{H}^{3} / \Gamma$. There exists a sequence $\left\{\left(r_{i}, k_{i}\right)\right\}$ and a sequence of maps $\tilde{f}_{i}: B_{r_{i}}(0) \rightarrow \mathbf{H}^{3}$ such that

1. $r_{i} \mapsto \infty$ and $k_{i} \mapsto 1$,

2. the map $\widetilde{f}_{i}$ is a $k_{i}$-biLipschitz diffeomorphism onto its image, $\widetilde{f}_{i}(0)=0$, and $\left\{\left.\tilde{f}_{i}\right|_{A}\right\}$ converges to the identity on any compact set $A$, and

3. $\widetilde{f}_{i}$ descends to an embedding $f_{i}: V_{i} \rightarrow N$ where $V_{i}=B_{r_{i}}(0) / \Gamma_{i}$ is a submanifold of $N_{i}$.

Proof of 3.6. Let $N_{i}=N_{\rho_{i}}$ and $N=N_{\rho}$, and let $\left\{f_{i}: V_{i} \rightarrow N\right\}$ be the sequence of biLipschitz embeddings given by Lemma 3.7. Proposition 3.3 in Canary-Minsky [CMi] implies that for all large enough $i,\left(f_{i}\right)_{*}$ is conjugate to $\rho \circ \rho_{i}{ }^{-1} \circ\left(j_{i}\right)_{*}$ where $j_{i}: V_{i} \rightarrow N_{i}$ is the inclusion map.

We first note that $N_{\delta}^{S} \cap C(N)$ has bounded diameter for all $\delta$, since $N$ is geometrically finite (see Bowditch [Bow].) We first choose $0<\epsilon^{\prime \prime}<\epsilon^{\prime}<\epsilon$ such that every closed geodesic $\gamma$ in $N$ either lies entirely in $N_{\epsilon^{\prime}}^{S}$ or entirely in $N_{\operatorname{thin}\left(\epsilon^{\prime}\right)}$, in which case we set $\gamma^{\prime}=\gamma$, or has a homotopic representative $\gamma^{\prime}$ lying entirely in $N_{\epsilon^{\prime \prime}}^{S} \cap C(N)$ all of whose segments are of length at least $L$ and are either geodesic subarcs of $\gamma$ ending in $\partial N_{\text {thin }\left(\epsilon^{\prime}\right)}$ or lie entirely in $\partial N_{\operatorname{thin}\left(\epsilon^{\prime}\right)}$ and are geodesics in the induced metric on $\partial N_{\operatorname{thin}\left(\epsilon^{\prime}\right)}$. (By choosing $\epsilon^{\prime}$ and $\epsilon^{\prime \prime}$ sufficiently small, we can make $L$ as large as we like.) Let $X$ be obtained from $N_{\epsilon^{\prime \prime}}^{S} \cap C(N)$ by appending every compact component of $N_{\operatorname{thin}\left(\epsilon^{\prime \prime}\right)}$. Then every representative $\gamma^{\prime}$ constructed as above lies in $X$.

We assume that we have chosen $i$ large enough so that $k_{i} \leq 2$ and $X \subset f_{i}\left(V_{i}\right)$. Given a closed geodesic $\gamma$ in $N$, let $\gamma_{i}$ denote the closed geodesic in $N_{i}$ in the homotopy class of $f_{i}^{-1}\left(\gamma^{\prime}\right)$. One shows using hyperbolic trigonometry, as in Taylor [T], that there exists $D>0$ such that for all large enough $i$ and all $\gamma, \gamma_{i}$ lies in $\mathcal{N}_{D}\left(f_{i}^{-1}\left(\gamma^{\prime}\right)\right) \cup\left(N_{i}\right)_{\operatorname{thin}(\epsilon)}$. For large enough $i$, every closed geodesic in $N_{i}$ either lies entirely in $\left(N_{i}\right)_{\operatorname{thin}(\epsilon)}$ or is homotopic to $f_{i}^{-1}\left(\gamma^{\prime}\right)$ for some closed geodesic $\gamma$ in $N$. Hence, every closed geodesic 
in $N_{i}$ lies in $\mathcal{N}_{D}\left(f_{i}^{-1}(X)\right) \cup\left(N_{i}\right)_{\text {thin }(\epsilon)}$ for all large enough $i$. Since there exists a uniform constant $E>0$ such that any point in the convex core of any hyperbolic 3-manifold lies within $E$ of a closed geodesic, it follows that $\left(N_{i}\right)_{\epsilon}^{S} \cap C\left(N_{i}\right)$ lies in $\mathcal{N}_{D+E}\left(f_{i}^{-1}(X)\right)$ for all large enough $i$. Since the diameter of $f_{i}^{-1}(X)$ is at most twice the diameter of $X$ for large enough $i$, it follows that $\left(N_{i}\right)_{\epsilon}^{S} \cap C\left(N_{i}\right)$ has diameter at most $2 \operatorname{diam}(X)+(D+$ $E)$ for all sufficiently large $i$. It is easy to check that $\left(N_{i}\right)_{\epsilon}^{S} \cap C_{K}\left(N_{i}\right)$ is contained in $\mathcal{N}_{2 K}\left(\left(N_{i}\right)_{\epsilon}^{S} \cap C\left(N_{i}\right)\right)$, so $\left(N_{i}\right)_{\epsilon}^{S} \cap C_{K}\left(N_{i}\right)$ has diameter at most $2 \operatorname{diam}(X)+D+E+2 K$ for all sufficiently large $i$.

\section{The Proof of Theorem 4.1}

Theorem 4.1. Let $M$ be a compact hyperbolizable 3-manifold. If $\left\{\rho_{i}\right\}$ is a sequence in $\mathcal{D}\left(\pi_{1}(M)\right)$ converging strongly to $\rho$ so that $\rho$ is geometrically finite. Then $\left\{\Lambda\left(\rho_{i}\right)\right\}$ converges to $\Lambda(\rho)$.

Proof of 4.1. The main theorem of Taylor [T] assures us that $\rho_{i}\left(\pi_{1}(M)\right)$ is geometrically finite for all large enough $i$. It is well known, see for example Lemma 5.2 in Canary-Minsky-Taylor [CMiT], that if $\left\{\Gamma_{i}\right\}$ converges geometrically to $\Gamma$, then $\lambda_{0}\left(\mathbf{H}^{3} / \Gamma\right) \geq \limsup \lambda_{0}\left(\mathbf{H}^{3} / \Gamma_{i}\right)$. Thus, $\Lambda(\rho) \geq \lim \sup \Lambda\left(\rho_{i}\right)$. We may pass to a subsequence $\left\{\rho_{j}\right\}$ of $\left\{\rho_{i}\right\}$ such that $\left\{\Lambda\left(\rho_{j}\right)\right\}$ converges to $L=\liminf \Lambda\left(\rho_{i}\right)$ and $\rho_{j}$ is geometrically finite for all $j$. We will prove that $\Lambda(\rho)=L$ which will suffice to establish the theorem. If $L=1$, then by upper semicontinuity of $\Lambda$ and the fact that $\Lambda(\rho) \leq \lambda_{0}\left(\mathbf{H}^{3}\right)=1$, we see that $\Lambda(\rho)=1$ and we are done. Thus, we may assume that $L<1$ and hence that $\Lambda\left(\rho_{j}\right) \leq 1-\delta_{1}<1$ for all sufficiently large $j$ and some $\delta_{1}>0$.

Let $N_{j}=N_{\rho_{j}}$ and $N=N_{\rho}$ and let $\left\{f_{j}: V_{j} \rightarrow N\right\}$ be the sequence of biLipschitz embeddings given by Lemma 3.7. Let $\phi_{j}$ be the normalized first eigenfunction associated to $\lambda_{0}\left(N_{j}\right)$ and let $p_{j}: \mathbf{H}^{3} \rightarrow N_{j}$ be the covering map. Choose $\mathcal{M}_{3}>\epsilon>0$. We may assume, without loss of generality, that $b_{j}=p_{j}(0) \in\left(N_{j}\right)_{\epsilon}^{S} \cap C\left(N_{j}\right)$ for all $j$.

Lemma 3.3 implies that there exists $C>0$ such that $\int_{\left(N_{j}\right)_{\epsilon}^{S}} \phi_{j}^{2} \geq C$ for all $j$. Lemma 3.5 implies that there exists $K>0$ such that $\int_{C_{K}\left(N_{j}\right)} \phi_{j}^{2} \geq 1-\frac{C}{2}$ for all $j$. Therefore,

for all $j$.

$$
\int_{C_{K}\left(N_{j}\right) \cap\left(N_{j}\right)_{\epsilon}^{S}} \phi_{j}^{2} \geq \frac{C}{2}
$$

Lemma 3.6 implies that there exists $A>0$ such that $C_{K}\left(N_{j}\right) \cap\left(N_{j}\right)_{\epsilon}^{S}$ 
has diameter less than $A$, for all sufficiently large $j$, and hence has volume less than the volume, say $V$, of the ball of radius $A$ in $\mathbf{H}^{3}$. Hence, for all sufficiently large $j$, there exists a point $x_{j} \in C_{K}\left(N_{j}\right) \cap\left(N_{j}\right)_{\epsilon}^{S}$ such that $\phi_{j}\left(x_{j}\right) \geq \sqrt{\frac{C}{2}} / V$.

Let $\left\{\widetilde{\phi}_{j}: \mathbf{H}^{3} \rightarrow \mathbf{R}\right\}$ denote the lifts of $\left\{\phi_{j}\right\}$ to $\mathbf{H}^{3}$. Yau's Harnack inequality implies that $\widetilde{\phi}_{j}(0)=\phi_{j}\left(b_{j}\right) \geq \frac{\sqrt{C / 2}}{V e^{R A}}$ for all sufficiently large $j$. Basic elliptic theory and Yau's Harnack inequality, then imply that a subsequence, still called $\left\{\widetilde{\phi}_{j}\right\}$, of $\left\{\widetilde{\phi}_{j}\right\}$ converges (uniformly on compact sets) to a non-zero, positive function $\widetilde{\phi}$ on $\mathbf{H}^{3}$ which is an eigenfunction for $-\Delta$ with eigenvalue $L$. Since $\left\{\rho_{j}\left(\pi_{1}(M)\right)\right\}$ converges geometrically to $\rho\left(\pi_{1}(M)\right), \widetilde{\phi}$ descends to a function $\phi$ on $N$. Since $\left\{\phi_{j} \circ f_{j}^{-1}\right\}$ converges (uniformly on compact sets) to $\phi$, it is easy to check that $\int_{N} \phi^{2} \leq 1$. Thus, $\phi$ is a multiple of the normalized first eigenfunction of $N$. Therefore, $\lambda_{0}(N)=\Lambda(\rho)=L$ which completes the proof.

Remarks. (1) The above proof can be easily generalized to obtain an analogous result in any dimension. It may also be generalized to yield McMullen's analogue of Theorem 4.1 which allows for a weaker notion of strong convergence.

(2) One might similarly ask if the discrete spectra of $N_{\rho_{i}}$ converge to the discrete spectrum of $N_{\rho}$. See Colbois-Courtois [CoCou], Chavel-Dodziuk $[\mathrm{ChD}]$ and $\mathrm{Ji}[\mathrm{J}]$ for related work on finite volume hyperbolic 3-manifolds.

\section{The Proofs of the Main Theorem and Corollaries}

We first use Theorem 4.1 to prove the Main Theorem.

Main Theorem. Let $M$ be a compact, hyperbolizable 3-manifold which is not homeomorphic to a handlebody. Then $D$ is continuous on $\mathcal{D}\left(\pi_{1}(M)\right)$ in the strong topology.

Proof of Main Theorem. First suppose that $M$ is not an $I$-bundle and that the sequence $\left\{\rho_{i}\right\} \subset \mathcal{D}\left(\pi_{1}(M)\right)$ converges to $\rho$ in the strong topology.

If $\rho$ is geometrically finite, then the main result of $[\mathrm{CT}]$ implies that $\Lambda(\rho) \neq 1$. Theorem 4.1 then implies that $\left\{\Lambda\left(\rho_{i}\right)\right\}$ converges to $\Lambda(\rho)$. One then applies Theorem 2.1 to see that $\left\{D\left(\rho_{i}\right)\right\}$ converges to $D(\rho)$.

If $\rho$ is not geometrically finite then $D(\rho)=2$ and $\operatorname{vol}(C(N))=\infty$. Since the volume of the convex core is a continuous function in the strong topology (see Taylor [T]) $\lim \operatorname{vol}\left(C\left(N_{i}\right)\right)=\infty$. (One may also argue more 
directly in the geometrically infinite setting, as in the proof of Lemma 7.1 in Canary-Minsky [CMi], that $\lim \operatorname{vol}\left(C\left(N_{i}\right)\right)=\infty$.) Theorem 2.2 then implies that $\left\{D\left(\rho_{i}\right)\right\}$ converges to 2 . Therefore, $D$ is continuous in the strong topology on $\mathcal{D}\left(\pi_{1}(M)\right)$ if $M$ is not an $I$-bundle.

Suppose that $M$ is an $I$-bundle over a closed surface and $\left\{\rho_{i}\right\}$ converges to $\rho$ in the strong topology. If $\rho$ is geometrically finite and $\Lambda(\rho)<1$, or $\rho$ is geometrically infinite, then the proof that $\left\{D\left(\rho_{i}\right)\right\}$ converges to $D(\rho)$ is just as above. If $\Lambda(\rho)=1$ and $\rho$ is geometrically finite, then the main result of Canary-Taylor [CT] implies that $\rho$ is convex cocompact. (In fact, $\rho\left(\pi_{1}(M)\right)$ contains a Fuchsian subgroup of index at most 2.) Marden's Stability Theorem $[\mathrm{M}]$ then implies that there is an open neighborhood $W$ of $\rho$ in $\mathcal{D}\left(\pi_{1}(M)\right)$ consisting of representations quasiconformally conjugate to $\rho$. It is then a standard consequence of results of Gehring and Väisälä [GV], see for example the proof of Corollary D in Canary-Minsky [CMi], that $D$ is continuous on $W$. (In fact, Corollary D in [CMi] implies immediately that $D$ is continuous on $W$.) In particular, $\left\{D\left(\rho_{i}\right)\right\}$ converges to $D(\rho)$ in this case as well. Therefore, $D$ is a continuous function in the strong topology of $\mathcal{D}\left(\pi_{1}(M)\right)$ if $M$ is an $I$-bundle over a closed surface.

Corollary A follows immediately from the Main Theorem and Theorem 2.1 if $M$ is not a handlebody. We will give a self-contained proof.

Corollary A. Let $M$ be a compact, hyperbolizable 3-manifold. Then $\Lambda$ is continuous on $T T\left(\pi_{1}(M)\right)$ in the strong topology.

Proof of Corollary A. Let $\left\{\rho_{i}\right\}$ be a sequence in $\mathcal{D}\left(\pi_{1}(M)\right)$ converging strongly to $\rho \in T T\left(\pi_{1}(M)\right)$. If $\rho$ is geometrically finite, then Theorem 4.1 implies immediately that $\left\{\Lambda\left(\rho_{i}\right)\right\}$ converges to $\Lambda(\rho)$. If $\rho$ is not geometrically finite, then it is shown in Canary [C] that $\Lambda(\rho)=0$. As above, this implies that $\lim \operatorname{vol}\left(C\left(N_{\rho_{i}}\right)\right)=\infty$. It then follows from the main result of Canary [C] that $\left\{\Lambda\left(\rho_{i}\right)\right\}$ converges to $\Lambda(\rho)$.

Corollary B follows from the Main Theorem and results of Bishop-Jones [BiJo] and Canary-Taylor [CT].

Corollary B. Let $M$ be a compact hyperbolizable 3-manifold which is not homeomorphic to a handlebody. Suppose that a sequence $\left\{\rho_{i}\right\}$ in $\mathcal{D}\left(\pi_{1}(M)\right)$ converges (in the compact-open topology) to $\rho \in \mathcal{D}\left(\pi_{1}(M)\right)$ such that $\rho$ is geometrically finite. Then $\left\{\rho_{i}\right\}$ converges strongly to $\rho$ if and only if $\left\{D\left(\rho_{i}\right)\right\}$ converges to $D(\rho)$.

Proof of Corollary B. If $\left\{\rho_{i}\right\}$ converges strongly to $\rho$, then it follows immediately from the Main Theorem that $\left\{D\left(\rho_{i}\right)\right\}$ converges to $D(\rho)$. 
In order to complete the proof we will suppose that $\left\{\rho_{i}\right\}$ does not converge strongly to $\rho$ and show that $\left\{D\left(\rho_{i}\right)\right\}$ does not converge to $D(\rho)$.

If $\rho_{i}$ is geometrically infinite for infinitely many $i$, then, by work of Bishop and Jones [BiJo], $D\left(\rho_{i}\right)=2$ for infinitely many $i$, so $\lim \sup D\left(\rho_{i}\right)=2$. However, since $\rho$ is geometrically finite, Sullivan [S1] and Tukia [Tu] showed that $D(\rho)<2$. So, in this case, $\left\{D\left(\rho_{i}\right)\right\}$ does not converge to $D(\rho)$.

We may now assume that $\rho_{i}$ is geometrically finite for all $i$. We first pass to a subsequence, $\left\{\rho_{j}\right\}$, so that $\left\{\rho_{j}\left(\pi_{1}(M)\right)\right\}$ converges geometrically to a torsion-free Kleinian group $\widehat{\Gamma}$ which contains $\rho\left(\pi_{1}(M)\right)$ as an infinite index subgroup (see Jørgensen-Marden $[\mathrm{J} \varnothing \mathrm{M}]$.) Notice that since $M$ is not a handlebody, the main result of Canary-Taylor [CT] implies that $D\left(\rho_{i}\right) \geq 1$ for all $i$ and that $D(\rho) \geq 1$. Lemma 5.2 in Canary-Minsky-Taylor [CMiT] implies that $\lambda_{0}(\widehat{N}) \geq \limsup \Lambda\left(\rho_{j}\right)$ where $\widehat{N}=\mathbf{H}^{3} / \widehat{\Gamma}$. Since $D(\rho) \geq 1$, Theorem 1 of Canary-Taylor [CT] implies that $\Lambda(\rho)>\lambda_{0}(\widehat{N})$. Hence, $\Lambda(\rho)>\lim \sup \Lambda\left(\rho_{j}\right)$. Since $\rho_{j}$ is geometrically finite for all $j$, Theorem 2.1 implies that $D(\rho)<\liminf D\left(\rho_{j}\right)$, which implies that $\left\{D\left(\rho_{j}\right)\right\}$ does not converge to $D(\rho)$ and hence that $\left\{D\left(\rho_{i}\right)\right\}$ does not converge to $D(\rho)$.

\section{A Conjecture}

There are many examples in which a sequence $\left\{\Gamma_{i}\right\}$ of Kleinian groups converges geometrically to a Kleinian group $\Gamma$, yet $\left\{\lambda_{0}\left(\mathbf{H}^{3} / \Gamma_{i}\right)\right\}$ does not converge to $\lambda_{0}\left(\mathbf{H}^{3} / \Gamma\right)$. (See, for instance, Taylor $[\mathrm{T}]$ or Comar-Taylor [ComT].) The easiest way to construct such examples is to let $\Theta$ be a fixed Kleinian group such that $\lambda_{0}\left(\mathbf{H}^{3} / \Theta\right) \neq 1$, and let $\gamma$ be an element of $\operatorname{PSL}_{2}(\mathbf{C})$ neither of whose fixed points lie in the limit set $L(\Theta)$. If we let $\Gamma_{j}=\gamma^{j} \Theta \gamma^{-j}$, then $\lambda_{0}\left(\mathbf{H}^{3} / \Gamma_{j}\right)=\lambda_{0}\left(\mathbf{H}^{3} / \Theta\right)$ for all $j$, but $\left\{\Gamma_{j}\right\}$ converges to the trivial group $\Gamma=\{1\}$, and $\lambda_{0}\left(\mathbf{H}^{3} / \Gamma\right)=1$. Moreover, in all the examples known to us there exists (up to subsequence) a sequence $\left\{\gamma_{i}\right\}$ of elements of $\mathrm{PSL}_{2}(\mathbf{C})$ such that $\left\{\gamma_{i} \Gamma_{i} \gamma_{i}^{-1}\right\}$ converges to a Kleinian group $\Gamma^{\prime}$ and $\left\{\lambda_{0}\left(\mathbf{H}^{3} / \Gamma_{i}\right)\right\}$ converges to $\lambda_{0}\left(\Gamma^{\prime}\right)$. This leads us to make the following conjecture.

Conjecture. Let $\left\{\Gamma_{i}\right\}$ be a sequence of Kleinian groups such that each $\Gamma_{i}$ is generated by at most $K$ elements. Then there exists a subsequence $\left\{\Gamma_{j}\right\}$ of $\left\{\Gamma_{i}\right\}$ and a sequence of elements $\left\{\gamma_{j} \in \mathrm{PSL}_{2}(\mathbf{C})\right\}$ such that $\left\{\gamma_{j} \Gamma_{j} \gamma_{j}^{-1}\right\}$ converges geometrically to $\Gamma$ and $\lambda_{0}\left(\mathbf{H}^{3} / \Gamma\right)=\lim \lambda_{0}\left(\mathbf{H}^{3} / \Gamma_{j}\right)$.

As evidence we notice that the argument used to establish Theorem 4.1, also establishes the above conjecture in the case that there exists a uniform 
bound on the diameter of $C\left(N_{i}\right) \cap\left(N_{i}\right)_{\epsilon}^{S}$ where $N_{i}=\mathbf{H}^{3} / \Gamma_{i}$.

\section{References}

[BPe] R. Benedetti, C. Petronio, Lectures on Hyperbolic Geometry, Springer-Verlag Universitext, 1992.

[BiJo] C. Bishop, P.W. Jones, Hausdorff dimension and Kleinian groups, Acta Math. 179 (1997), 1-39.

[Bo] F. Bonahon, Bouts des variétés hyperboliques de dimension 3, Annals of Math. 124 (1986), 71-158.

[Bow] B.H. BowDitch, Geometrical finiteness for hyperbolic groups, J. Func. Anal. 113 (1993), 245-317.

[BuC] M. Burger, R.D. CAnary, A lower bound for $\lambda_{0}$ for geometrically finite hyperbolic $n$-manifolds, J. reine angew. Math. 454 (1994), 37-57.

[C] R.D. Canary, On the Laplacian and the geometry of hyperbolic 3manifolds, J. Diff. Geom. 36 (1992), 349-367.

[CEGr] R.D. Canary, D.B.A. Epstein, P. Green, Notes on notes of Thurston, in "Analytical and Geometrical Aspects of Hyperbolic Spaces," Cambridge University Press (1987), 3-92.

[CMi] R.D. Canary, Y.N. Minsky, On limits of tame hyperbolic 3-manifolds, J. Diff. Geom. 43 (1996), 1-41.

[CMiT] R.D. Canary, Y.N. Minsky, E.C. Taylor, Spectral theory, Hausdorff dimension and the topology of hyperbolic 3-manifolds, Journal of Geometric Analysis, to appear.

[CT] R.D. CANARY, E.C. TAYLOR, Kleinian groups with small limit sets, Duke Math. J. 73 (1994), 371-381.

[ChD] I. Chavel, J. Dodziuk, The spectrum of degenerating hyperbolic 3manifolds, J. Diff. Geom. 39 (1994), 123-137.

[CoCou] B. Colbois, G. Courtois, Convergence de varietes et convergence du spectre du Laplacien, Ann. Sci. École Norm. Sup. 24 (1991), 507-518.

[ComT] T. Comar, E.C. TAYlOR, Geometrically convergent Kleinian groups and the lowest eigenvalue of the Laplacian, Indiana Math. J. 47 (1998), $601-623$.

[DR] J. Dodziuk, B. Randol, Lower bounds for $\lambda_{1}$ on a finite volume hyperbolic manifold, J. Diff. Geom. 24 (1986), 133-139.

[EM] D.B.A. Epstein, A. Marden, Convex hulls in hyperbolic spaces, a theorem of Sullivan, and measured pleated surfaces, in "Analytical and Geometrical Aspects of Hyperbolic Spaces", Cambridge University Press (1987), 113-253.

[GV] F.W. GEhring, J. VÄIsÄLÄ, Hausdorff dimension and quasiconformal mappings, Journal of the L.M.S. 6 (1973), 504-512.

$[\mathrm{J}] \quad$ L. JI, Spectral convergence for degenerating sequences of three- 
dimensional hyperbolic manifolds, Trans. Amer. Math. Soc. 348 (1996), 2673-2688.

[JøM] T. Jørgensen, A. MARDEn, Algebraic and geometric convergence of Kleinian groups, Math. Scand. 66 (1990), 47-72.

[LPh] P. LAx, R.S. Phillips, The asymptotic distribution of lattice points in Euclidean and non-Euclidean spaces, J. Funct. Anal. 46 (1982), 280-350.

[M] A. Marden, The geometry of finitely generated Kleinian groups, Annals of Math. 99 (1974), 383-462.

[Mc] C.T. McMullen, Hausdorff dimension and conformal dynamics I: Strong convergence of Kleinian groups, preprint.

[P] S.J. Patterson, Lectures on limit sets of Kleinian groups, in "Analytical and Geometrical Aspects of Hyperbolic Spaces," Cambridge University Press (1987), 281-323.

[S1] D. Sullivan, Entropy, Hausdorff measures old and new, and limit sets of geometrically finite Kleinian groups, Acta. Math. 153 (1984), 259-277.

[S2] D. Sullivan, Aspects of positivity in Riemannian geometry, J. Diff. Geom. 25 (1987) 327-351.

[T] E.C. TAYLOR, Geometric finiteness and the convergence of Kleinian groups, Communications in Analysis and Geometry, 8 (1997), 497-533.

[Tu] P. Tukia, The Hausdorff dimension of the limit sets of geometrically finite Kleinian groups, Acta Math. 152 (1984), 127-140.

[Y] S.T. YAU, Harmonic functions on complete Riemannian manifolds, Comm. Pure Appl. Math. 28 (1975), 201-228.

Richard D. Canary and Edward C. Taylor

Department of Mathematics

University of Michigan

Ann Arbor, MI 48109C

USA

canary@math.lsa.umich.edu

ectaylor@math.lsa.umich.edu

Submitted: January 1998 